\title{
Decelerated aging in metallic glasses by low temperature thermal cycling
}

\author{
Marian Bruns $\odot,{ }^{*}$ Muhammad Hassani, ${ }^{\dagger}$ and Fathollah Varnik ${ }^{\ddagger}$ \\ Interdisciplinary Centre for Advanced Materials Simulation (ICAMS) and Ruhr-Universität Bochum, \\ Universitätsstraße 150, 44801 Bochum, Germany \\ Afrouz Hassanpour, ${ }^{\S}$ Sergyi Divinski $\odot, "$ and Gerhard Wilde $\oplus^{\mathbb{I}}$ \\ Institut für Materialphysik and Universität Münster, Wilhelm-Klemm-Straße 10, 48149 Münster, Germany
}

(Received 15 October 2020; revised 8 December 2020; accepted 15 December 2020; published 12 March 2021)

\begin{abstract}
Differential scanning calorimetry measurements on different bulk metallic glasses show no measurable rejuvenation upon deeply cooled (cryogenic) thermal cycling. This applies both to as-quenched and wellannealed samples. Extensive molecular dynamics simulations of a generic model glass former corroborate these observations. We disentangle the effects of aging from those of thermal treatment and show that aging is slowed down but not stopped-neither reversed-during thermal cycling. These observations are corroborated further by a survey of energy distribution, which continues narrowing, albeit with a smaller rate.
\end{abstract}

DOI: 10.1103/PhysRevResearch.3.013234

\section{INTRODUCTION}

Aging of glasses at temperatures well below the glass transition is marked by the evolution towards an unreachable equilibrium state. This physical aging [1] process leads to a slowing down of the relaxation dynamics [2,3] and narrowing of the energy distribution [4]. Well-aged glasses show low ductility and reduced plastic deformability [5-9], hampering their application as structural materials.

Recently, it has been proposed that thermal cycling (TC)also referred to as (deep) cryogenic cycling-can induce rejuvenation effects, thereby improving the ductility of bulk metallic glasses (BMGs) [10-13]. A possible mechanism for this effect has been proposed by Hufnagel [14] in terms of the spatial heterogeneity of the thermal expansion coefficient, thus, cyclic cooling and reheating, leading to internal stresses, enhances structural disorder and leads to a softening of the material [10].

The potential of heterogeneous internal strain to account for rejuvenation effects has been scrutinized in recent computer simulation studies of the thermal expansion coefficient and its spatial fluctuations in a $\mathrm{Cu}_{50} \mathrm{Zr}_{50}$ model glass [15]. Another molecular dynamics (MD) study of a binary LennardJones (LJ) mixture [16] implies that annealing treatments

\footnotetext{
*marian.bruns@rub.de

†sayed.hassani@rub.de

‡Corresponding author: fathollah.varnik@rub.de

§hassanpa@uni-muenster.de

"divin@uni-muenster.de

Igwilde@uni-muenster.de
}

Published by the American Physical Society under the terms of the Creative Commons Attribution 4.0 International license. Further distribution of this work must maintain attribution to the author(s) and the published article's title, journal citation, and DOI. can have a strong impact on the rejuvenation behavior, with "poorly" annealed glasses revealing continuous aging, while "well-annealed" ones show rejuvenation.

However, while first experiments on $\mathrm{Cu}_{46} \mathrm{Zr}_{46} \mathrm{Al}_{7} \mathrm{Gd}_{1}$ and $\mathrm{La}_{55} \mathrm{Ni}_{10} \mathrm{Al}_{35}$ glasses showed indication of cryogenic cycling-induced rejuvenation [10,17], later studies revealed unexpected subtleties. Experiments on Pd-based samples, for example, showed no obvious signature of rejuvenation effects upon deep thermal cycling [18]. Moreover, and in contrast to Ref. [16], it was also reported in Ref. [18] that well-annealed Zr-based BMGs do not show any detectable rejuvenation.

The present paper sets in at this point. We first perform calorimetric investigations of $\mathrm{PdNiP}$ and $\mathrm{Zr}$-based glasses both "as-quenched" and "annealed" with a focus on possible effects of deep TC. The two Pd-Ni-based bulk metallic glasses with a slightly varying composition represent wellknown model cases for metal-metalloid glasses [19-23] while the Zr-based BMG is a model system for purely metallic glasses [24].

To the best of our knowledge, no experiments were performed on cryo-cycling of PdNiP glass. On the other hand, there exist reports on Zr-based glasses, but of different compositions compared to that used in the present work [17,25,26]. Our experiments show no measurable changes in the heat release during repeated cooling and reheating of the investigated BMGs after a relatively large number of cycles.

One of central goals of the present study is the fundamental question whether rejuvenation-documented for some metallic glasses and not observed for some others-is a generic property of a bulk metallic glass. The two BMG systems, namely, PdNiP- and Zr-based ones, are chosen as their behavior is well understood, and a wealth of experimental data is accumulated. These are fundamentally two distinct BMG families even with respect to their glass-forming ability. The PdNiP glass represents an almost ideal glass in which nucleation of a crystalline phase can effectively be bypassed during 
slow casting [19]. On the other hand, macroscopically large Zr-based glass can be produced since diffusion in this system is strongly suppressed (nevertheless its atomistic structure is characterized by a relatively large number density of crystal nuclei [24]). The fact that three different experimental probes in our study - as a third system we have chosen a PdNiP glass with a nonideal composition-show a similar calorimetric behavior suggests that there is probably a trend behind the observed lack of measurable rejuvenation.

In order to provide an ultimate understanding of the propensity of such good glass formers to rejuvenation from molecular dynamics perspective, most general interatomic potentials have to be chosen. This motivates us to choose a model which is as generic as possible, while at the same time being not too much unrealistic. The present (80:20) binary LJ glass, first proposed by Kob and Anderson [27], is such a candidate as it has been successfully employed to investigate various aspects of the glass transition both in the quiescent state [28-32] as well as under mechanical deformation [33-36]. Moreover, it has also been utilized to mimic properties of $\mathrm{Ni}_{80} \mathrm{Pd}_{20}$ [37].

The absence of a net overall rejuvenation in our experiments is corroborated by molecular dynamics simulations via a survey of the structural relaxation and the evolution of energy distribution. A direct comparison of relaxation dynamics with and without thermal cycling reveals that (1) physical aging is present in both cases and (2) it is slower during TC.

The present paper is organized as follows. In the next section, we briefly describe the experiments and present the thus obtained results. Section III is devoted to molecular dynamics simulations. Section IV provides a summary and an outlook for future studies.

\section{EXPERIMENTS}

We perform differential scanning calorimetry (DSC) measurements on a number of bulk metallic glasses to investigate the effect of deep thermal cycling on materials properties. After a brief description of the sample preparation procedure, DSC results are discussed with a focus on possible rejuvenation effects.

\section{A. Sample preparation}

The $\mathrm{Pd}_{40} \mathrm{Ni}_{40} \mathrm{P}_{20}$ and $\mathrm{Pd}_{39} \mathrm{Ni}_{41} \mathrm{P}_{20}$ (in at.\%) bulk metallic glasses were produced by copper mold casting under argon atmosphere using a melt spinning device. To purify the sample, the ingots were cycled with boron oxide $\left(\mathrm{B}_{2} \mathrm{O}_{3}\right)$. The melt of palladium (purity $99.95 \mathrm{wt} \%$ ) and $\mathrm{Ni}_{2} \mathrm{P}(99.5$ wt.\%) was blown out of fused silica crucible and cooled with a rate of $100 \mathrm{~K} / \mathrm{min}$ into the copper mold. The temperature of casting for PdNiP glasses was controlled to be about $900 \pm 20^{\circ} \mathrm{C}$.

Master alloy ingots with the nominal composition $\mathrm{Zr}_{41.2} \mathrm{Be}_{22.5} \mathrm{Ti}_{13.8} \mathrm{Cu}_{12.5} \mathrm{Ni}_{10}$ (Vitreloy 1) were prepared by arc-melting the elements $\mathrm{Zr}, \mathrm{Ti}, \mathrm{Cu}, \mathrm{Ni}$, and $\mathrm{Be}$ (with a purity of $99.9 \%$ or better) together in a Ti-gettered highpurity argon atmosphere in the research group of Prof. M.-Q. Jiang at the Institute of Mechanics, Chinese Academy of Science, Beijing, China. To ensure homogeneity, the ingots were remelted several times and subsequently suction-drawn into copper molds to form plates $\left(100 \times 20 \times 2 \mathrm{~mm}^{3}\right)$. The material has been examined carefully by XRD for the absence of any detectable crystalline fraction. The samples of several $\mathrm{mm}$ in diameter were cut by spark erosion from the center parts of the materials. The temperature measurements during casting are unreliable due to the local heating with the electron beam and the uncontrolled cooling through heat losses at the copper/alloy interface and the alloy surface. Yet, due to the comparably high surface tension, the alloy needs to be heated to temperatures well above its liquidus point in order to perform appropriate suction casting.

The thermal cycling between room and cryogenic temperatures was done on both as-cast and preannealed samples. Typically cube-shaped samples of $2 \times 2 \times 2 \mathrm{~mm}^{3}$ to $3 \times 3 \times$ $3 \mathrm{~mm}^{3}$ in size were used. Note that no influence of this size variation on the calorimetric data was found. This observation underlines the correctness of the chosen procedure. The samples were placed in a metal cage and inserted into liquid nitrogen (temperature of $77 \mathrm{~K}$ ) for $60 \mathrm{~s}$ and then were taken out and heated fast to room temperature using a blow dryer for another $60 \mathrm{~s}$. This procedure, which defines one full cycle, was repeated the given number of times.

The impact of preannealing was investigated on the $\mathrm{Pd}_{40} \mathrm{Ni}_{40} \mathrm{P}_{20}$ glass by fast heating the samples in the DSC device to the selected temperatures $(473 \mathrm{~K}$ and $523 \mathrm{~K}$, heating rate $20 \mathrm{~K} / \mathrm{min}$ ) and holding them for $1 \mathrm{~h}$ followed by rapid cooling with the rate of $300 \mathrm{~K} / \mathrm{min}$.

Differential scanning calorimetry analysis was performed using a PerkinElmer Diamond device. The DSC measurements were performed on both as-cast and as-cast plus thermally cycled samples in the temperature range of $323 \mathrm{~K}$ to $798 \mathrm{~K}$ using the same heating and cooling rate of $20 \mathrm{~K} / \mathrm{min}$.

\section{B. Experimental results}

Figure 1(a) shows the results of DSC measurements for as-cast and as-cast plus thermally cycled (TC) $\mathrm{Pd}_{40} \mathrm{Ni}_{40} \mathrm{P}_{20}$ bulk metallic glass. The heat release near the glass transition temperature, $T_{\mathrm{g}}$, is explicitly shown for a better comparison of the relevant changes induced by cryogenic cycling. As seen from this plot, the calorimetry data show only a weak difference between the heat release of as-cast and as-cast+TC samples. However, this difference is even smaller than the typical experimental uncertainties estimated at $\pm 0.07 \mathrm{~J} / \mathrm{mol} \mathrm{K}$ (indicated in the plot) and shall not be overinterpreted. Similar results are obtained for the PdNiP glass of a slightly different composition, the $\mathrm{Pd}_{39} \mathrm{Ni}_{41} \mathrm{P}_{20}$ glass [Fig. 1(b)] and Zr-based Vitreloy 1 [Fig. 1(c)].

Furthermore, the cryogenic cycling was performed on the $\mathrm{Pd}_{40} \mathrm{Ni}_{40} \mathrm{P}_{20}$ as-cast samples after their preannealing (relaxation) at $473 \mathrm{~K}$ and $523 \mathrm{~K}$ for $1 \mathrm{~h}$, i.e., reasonably below the glass transition temperature; see Fig. 2 [38]. Again, DSC measurements do not reveal any significant difference between the heat fluxes in the given states. Twenty cycles of cryogenic treatment of the sample relaxed at $473 \mathrm{~K}$ do not introduce any measurable difference in comparison to the as-cast state in terms of the heat release [Fig. 2(a)]. Similarly, the DSC measurements confirm that the influence of the cryogenic cycling on the heat release remains within typical experimental uncertainties in the sample relaxed previously at $523 \mathrm{~K}$ even up to 40 cycles [Fig. 2(b)]. As shown in Fig. 2(b), the 

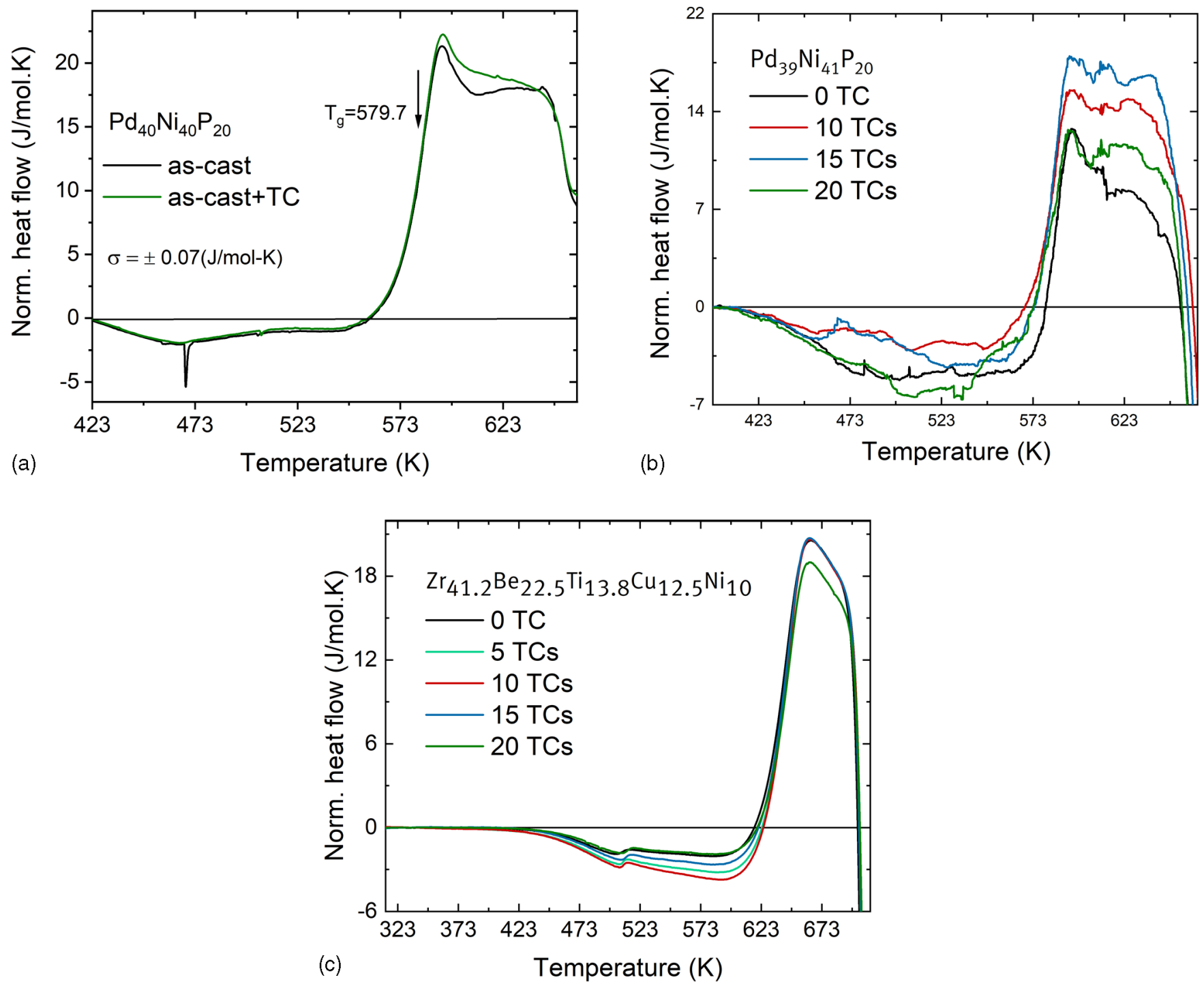

FIG. 1. DSC signals of $\mathrm{Pd}_{40} \mathrm{Ni}_{40} \mathrm{P}_{20}$ (a), $\mathrm{Pd}_{39} \mathrm{Ni}_{41} \mathrm{P}_{20}$ (b), and $\mathrm{Zr}_{41.2} \mathrm{Be}_{22.5} \mathrm{Ti}_{13.8} \mathrm{Cu}_{12.5} \mathrm{Ni}_{10}$ (c) bulk metallic glasses after different numbers of thermal cycles in comparison to the corresponding as-cast states. A typical experimental uncertainty of heat flow measurements, $\sigma= \pm 0.07$ $\mathrm{J} / \mathrm{mol} \mathrm{K}$, is about the line thickness. Positive heat flux indicates endothermic signal.

reproducibility of the results has been verified performing DSC measurements on different pieces of the original ingots (noted as $\mathrm{S} X, X=1, \ldots, 9$ ). For all the glasses investigated in this work, we have determined the relaxation enthalpy, $\Delta H_{\text {rel }}$, as the area on the (base-line subtracted) heat-flow curve from the onset of relaxation to the glass transition [21,39]. The results are presented in Fig. 3.

In the $\mathrm{Pd}_{39} \mathrm{Ni}_{41} \mathrm{P}_{20}$ glass, the relaxation enthalpy first decreases from $0.58 \mathrm{~kJ} / \mathrm{mol}$ for the as-cast glass to about $0.29 \mathrm{~kJ} / \mathrm{mol}$ after 10 cycles and then increases again to $0.39 \mathrm{~kJ} / \mathrm{mol}$ after 15 cycles and approaches the initial level of $0.63 \mathrm{~kJ} / \mathrm{mol}$ after 20 cycles. Thus, some aging behavior after a low number of cycles might be present in this case.

Thermo-cycling of the Zr-based glass [40] increases the relaxation enthalpy from $0.26 \mathrm{~kJ} / \mathrm{mol}$ for the as-cast state to $0.41 \mathrm{~kJ} / \mathrm{mol}$ and $0.45 \mathrm{~kJ} / \mathrm{mol}$ after five and 10 cycles, respectively. After 15 cycles, however, $\Delta H_{\text {rel }}$ decreases to 0.32 $\mathrm{kJ} / \mathrm{mol}$. Upon an additional five cycles, i.e., 20 cycles in total, a further decrease is observed to $0.25 \mathrm{~kJ} / \mathrm{mol}$, the value measured also in the as-cast state. Indeed, an increase of $\Delta H_{\text {rel }}$ in Zr-based glass by about $0.2 \mathrm{~kJ} / \mathrm{mol}$ in the first 10 cycles of our experiments (Fig. 3) is similar to that reported by Ketov et al. [10], albeit for a different system, $\mathrm{La}_{55} \mathrm{Ni}_{10} \mathrm{Al}_{35}$ metallic glass. However, it is also visible in Fig. 3 that $\Delta H_{\text {rel }}$ decreases back to its as-cast value upon further cycling. The rejuvenation effect associated with the initial increase of $\Delta H_{\text {rel }}$ thus seems to be reversed upon increasing the number of cycles in $\mathrm{Zr}$-based glass.

Let us also mention that, in the original work of Ketov et al. [10], noticeable rejuvenation was observed only for ribbons (see also results of Das et al. [41] on a comparison of rejuvenation in Zr-based melt-spun and cast glasses). However, as also reported in Ref. [10], cryogenic thermal cycling shows only minor effects on bulk samples which we focus upon in the present study.

Moreover, it is noteworthy that Zr-based Vitreloy 1 cannot be treated as a generic model system, due to the tendency 

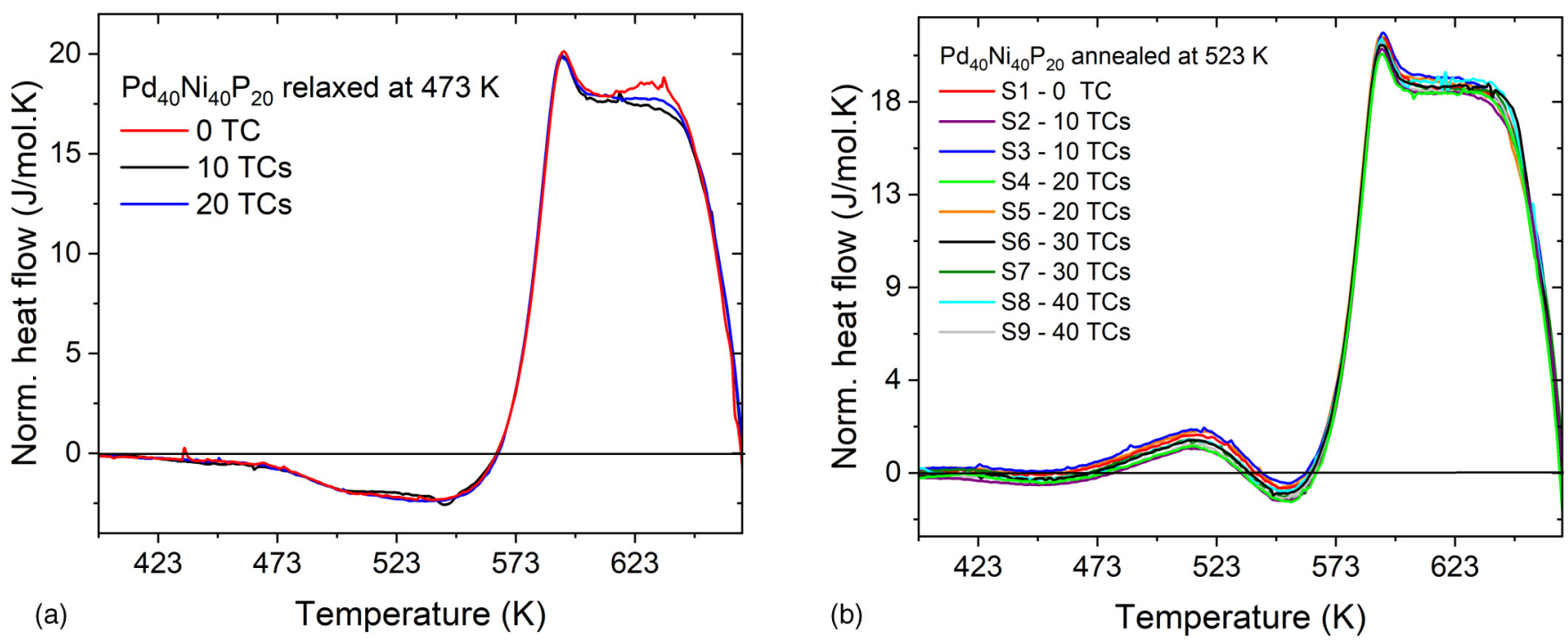

FIG. 2. The impact of a preannealing heat treatment at $473 \mathrm{~K}$ (a) and $523 \mathrm{~K}$ (b) on the DSC signal in $\mathrm{Pd}_{40} \mathrm{Ni}_{40} \mathrm{P}_{20}$ bulk metallic glass after different numbers of thermal cycles. The designations S1 to S9 correspond to samples taken from different locations of the same ingot or even from different ingots of the same compositions. Positive heat flux indicates endothermic signal.

for phase separation and (nano)crystallization in the deeply undercooled liquid $[42,43]$. This places $\mathrm{Zr}$-based glasses in a specific rather than a generic category. In contract, nucleation (or phase separation) can be completely avoided for the Pdbased model glass $\left(\mathrm{Pd}_{40} \mathrm{Ni}_{40} \mathrm{P}_{20}\right)$.

With respect to their glass-forming abilities, the BMGs chosen in the present study are relatively similar to those investigated by Ketov et al. [10]. Glass heterogeneity, however, is a more intricate issue.

Here one has to distinguish between different levels of heterogeneity regarding properties inherent to any glassy material:

(1) On a macroscopic scale, a heterogeneity of composition and structure can be induced by differences in local cooling rates and thermal gradients. In all our experiments, we

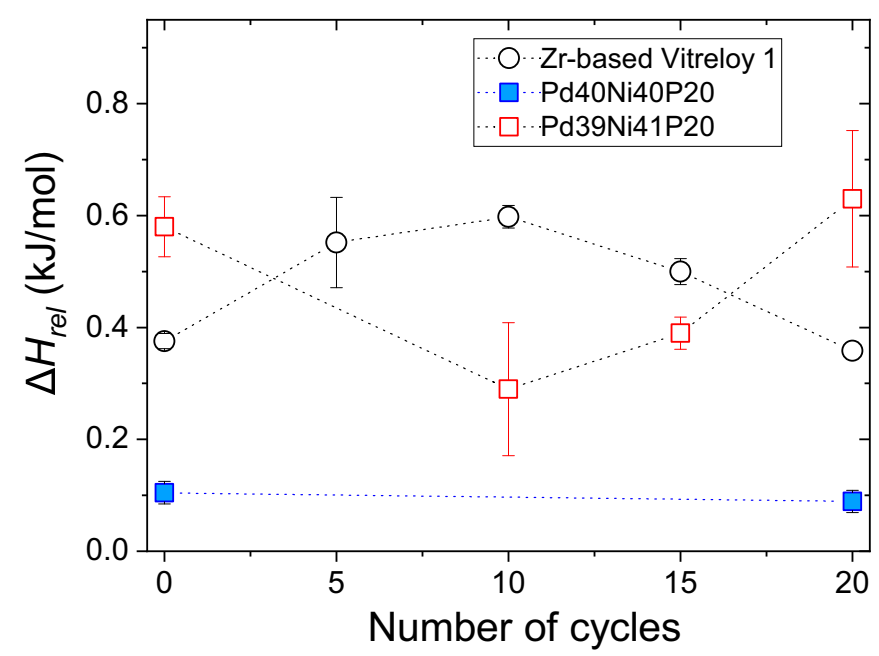

FIG. 3. Heat of relaxation as a function of the number of thermocycles for PdNiP (two compositions, squares) and Zr-based Vitreloy 1 (circles) glasses. paid attention that the samples are taken from representative and adequately characterized positions of cast blocks (far from external surfaces). Experimentally, slight (sometimes inevitable) deviations in composition of, e.g., PdNiP glass from the ideal 40:40:20 ratio were found to result in moderate changes of $T_{\mathrm{g}}, T_{\mathrm{X}}$ (the onset temperature of crystallization), or $\Delta H$; see, e.g., Ref. [21]. Thus, it is imperative to characterize the thermodynamic properties of glasses with a full account of their composition, as it was done in the present study.

(2) On the other hand, there is an intrinsic heterogeneity of local motifs, atomic configurations, excess free volume, etc., in the given glass (of the given composition). Recent measurements have shown that different glasses are characterized by different fractions of medium range order (MRO) motifs whose occurrence and spatial distribution can be affected by thermo-mechanical processing [24]. These local heterogeneities are in fact a source of potential changes which might be induced in the glass by cryo-cycling. The present experimental results show that there is no systematic variation of the heat release below $T_{\mathrm{g}}$ which could be treated as an indication of a rejuvenation behavior in PdNiP glass, while such a tendency may be stated for the Zr-based glasses. It is known now that these glasses posses different MROs with probably different heterogeneities of their distributions. How far the presence or absence of rejuvenation can be considered as a generic property of a BMG, has to be elucidated using atomistic simulations for an "ideal" glass.

The different behaviors observed for the two glass families could be a consequence of different characteristic times for Pd-based and Zr-based glasses. The Zr-based glass shows probably some rejuvenation at first cycles since aging in this system occurs on a time scale which is long compared to the cycling period. Only after increasing the free enthalpy during initial cycling (or accumulating sufficient internal stress levels), aging dynamics becomes faster and can proceed on a similar timescale as in our experiments. 
In view of the formidable experimental challenges associated with such detailed investigations, molecular dynamics simulations appear very promising here as they naturally provide full information on all these quantities [15]. Below we follow this route and investigate via molecular dynamics simulations the behavior of a model glass under conditions of deep temperature cycling.

\section{MODEL AND SIMULATION PROTOCOL}

As mentioned above, the (80:20) binary LJ model glass used in the present work has been first proposed by Kob and Andersen and has proved to be quite successful in addressing the glass transition phenomena. It has also been used to investigate, on a qualitative level, properties of $\mathrm{Ni}_{80} \mathrm{Pd}_{20}$, which is a metal-metalloid glass. Here we provide some details of this model.

Interactions in the present model are given by the binary $\mathrm{LJ}$ potential, which acts between particle pairs of types $\alpha$ and $\beta$ via $U_{\mathrm{LJ}}(r)=4 \varepsilon_{\alpha \beta}\left[\left(\sigma_{\alpha \beta} / r\right)^{12}-\left(\sigma_{\alpha \beta} / r\right)^{6}\right]$ with $\alpha, \beta=\mathrm{A}$, B. For the parameters we chose $\varepsilon_{\mathrm{AA}}=1, \varepsilon_{\mathrm{AB}}=1.5, \varepsilon_{\mathrm{BB}}=0.5$, $\sigma_{\mathrm{AA}}=1, \sigma_{\mathrm{AB}}=0.8, \sigma_{\mathrm{BB}}=0.88$, and $m_{\mathrm{A}}=m_{\mathrm{B}}=1 . \varepsilon_{\mathrm{AA}}$, $\sigma_{\mathrm{AA}}$, and $m_{\mathrm{A}}$ define the units of energy, length, and mass. The unit of time is then given by $\tau_{\mathrm{LJ}}=\sigma_{\mathrm{AA}} \sqrt{m_{\mathrm{A}} / \varepsilon_{\mathrm{AA}}}$ and the unit of temperature by $T_{\mathrm{LJ}}=\varepsilon_{\mathrm{AA}} / k_{\mathrm{B}}$. The potential is truncated at the cutoff radius $r_{\mathrm{c}}=2.245$ being twice the minimum range of $U_{\mathrm{LJ}}$ to reduce computational costs. At a fixed total number density of $\rho=1.2$, the system exhibits a computer glass transition at $T_{\mathrm{g}} \approx 0.41[34,44]$.

In addition to the considerations mentioned in the introduction regarding its generic character and reliability, the choice of this model is further motivated by our familiarity with various aspects of its behavior such as nonlinear rheology and shear banding [33,35,45], effects of aging on response to external shear [34], structural heterogeneity upon shear $[36,46]$ and long-range strain correlations $[47,48]$.

Two system sizes are considered here, which differ by roughly a factor of 70 in particle number. This allows to examine the possible effects of the system size on rejuvenation under cryogenic thermal cycling [15]. While for computational reasons most of the simulations are performed for a relatively small box size of $L \approx 24$, containing $N=16384$ particles, a number of representative studies are repeated for a large box of $L=100$ with $N=1203052$ particles. Periodic boundary conditions are applied in all the spatial directions. Equations of motion are integrated using the software package LAMMPS [49] with a discrete time step of $d t=0.005 \tau_{\mathrm{LJ}}$. Temperature is controlled via the Nosé-Hoover thermostat [50]. Whenever constant-pressure ( $N p T$-) simulations are performed, pressure is kept constant using the Parrinello-Rahman barostat [51].

Due to the lack of ergodicity at temperatures considered in this study, we have paid special attention to prepare samples with a controlled history. Figure 4 illustrates the protocol used in this study. First, the system is equilibrated at a relatively high temperature of $T=2.0$ (liquid state) for a duration of $t=1000 \tau_{\mathrm{LJ}}$. This time is roughly 200 times larger than the structural $\alpha$-relaxation time (defined here as the time needed for an A-particle to move a distance comparable to its own size). We also recall here that B-particles are more mobile than

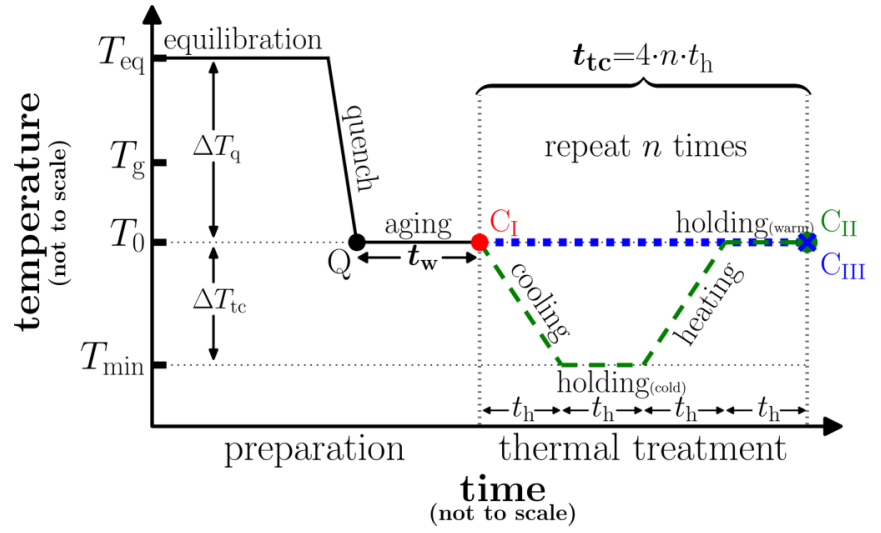

FIG. 4. Schematic representation of the preparation protocol and thermal treatment. The system is first equilibrated in the liquid state at a relatively high temperature of $T=2.0$ for a duration of $t=$ $1000 \tau_{\mathrm{LJ}}$. It then is quenched to a low temperature, $T_{0}<T_{\mathrm{g}} \approx 0.41$ [44], where it falls out of equilibrium (point $Q$ ). The system is evolved at this temperature for a duration of $t_{\mathrm{w}}$. At this point, three identical copies of particle positions and velocities are created. One of these configuration-copies is kept unchanged (sample " $\mathrm{C}_{\mathrm{I}}$ "). The second copy is thermally cycled between $T_{0}$ and $T_{\min }$ employing 10 full cycles with a total duration of $t_{\mathrm{tc}}$. This brings the system to a configuration called " $\mathrm{C}_{\mathrm{II}}$." The third copy of the system configuration is subjected to aging during $t_{\mathrm{tc}}$ without thermal cycling (sample $\mathrm{C}_{\mathrm{III}}$ ).

A-ones [28]. The system is then quenched instantaneously to a temperature $T_{0}$ which lies well below the glass transition point, $T_{0}<T_{\mathrm{g}}$.

As $T_{0}$ is reached (point $\mathrm{Q}$ ), cooling is stopped and the system is let to evolve with time for a duration of $t_{\mathrm{w}}$. This allows to account for the aging process, which takes place between the quench to a glassy state and the beginning of thermal cycling. There is a lower bound to $t_{\mathrm{w}}$ in MD simulations, related to the fast "microscopic" dynamics. From previous studies of the model under steady shear, we know that the time scale of these processes is of the order of a few hundred LJ time units [45]. Therefore, we set the $t_{\mathrm{w}} \geqslant 500 \tau_{\mathrm{LJ}}$ in this study. All the simulations until point $\mathrm{C}_{\mathrm{I}}$ are performed at constant volume $(N V T)$.

After the initial aging step, three identical copies of the entire system configuration including particle positions and velocities are created. One of these copies is subjected to oscillatory temperature variations between $T_{0}$ and $T_{\min }$ at constant pressure for a number $n$ of full cycles. The pressure applied in this stage is the time average of the internal pressure after being aged for $t_{\mathrm{w}}=50000 \tau_{\mathrm{LJ}}$. Given the duration $4 t_{\mathrm{h}}$ of a thermal cycle (Fig. 4), the total duration of the cycling process reads $t_{\mathrm{tc}}=4 n t_{\mathrm{h}}$. The endpoint of this process defines the configuration called $\mathrm{C}_{\mathrm{II}}$. From this point on, all the simulations are again performed in the $N V T$ ensemble at a constant temperature of $T_{0}$. This thermal cycling mimics the experimental conditions, although the computer times and temperatures have not to be directly translated to the experimentally accessible quantities. As was stated in the introduction, we are interested in a generic behavior and the impact of cryogenic thermal cycling on basic properties of a bulk metallic glass. 
It is noteworthy that the use of $N p T$ ensemble during cryogenic cycling is motivated by the wish to be as close as possible to experiments, which are usually performed under constant ambient pressure rather than constant sample volume. After thermal cycling, however, simulating at constant pressure is not an absolute necessity and other alternatives can be chosen as well. Since in simulations dynamic quantities are sensitive to fluctuations of simulation cell size [52], all measurements of the relaxation dynamics reported in this work are performed at constant volume ( $N V T$ ensemble).

The second copy of the starting configuration is not thermally cycled but continues its time evolution without any perturbation until it reaches point $\mathrm{C}_{\mathrm{III}}$. The sample denoted as $\mathrm{C}_{\mathrm{III}}$ has thus been subject to physical aging process during a total time of $t_{\mathrm{w}}^{\prime}=t_{\mathrm{w}}+t_{\mathrm{tc}}$.

The main motivation for the above choice of protocol is the following. A comparison of the dynamics starting at point $C_{I}$ and $\mathrm{C}_{\mathrm{II}}$ will reveal whether the dynamics continues to slow down despite thermal cycling. A comparison of dynamics measurements starting with configurations $\mathrm{C}_{\mathrm{II}}$ and $\mathrm{C}_{\mathrm{III}}$, on the other hand, will allow to decide whether and how the aging process is influenced by thermal cycling.

We will see that a sample subject to low temperature thermal cycling exhibits a faster relaxation dynamics as compared to a sample which undergoes aging alone. This can be interpreted either as rejuvenation or as decelerated aging. A comparison between samples $\mathrm{C}_{\mathrm{I}}$ and $\mathrm{C}_{\mathrm{II}}$ then reveals that some degree of aging is present during cryogenic cycling. Putting these two pieces of information together, one can then conclude that, within the present study, thermal cycling slows down the aging process but does not overcome it. We discuss these findings with a special attention to the possible role of small system size in our simulations as suggested by Barrat and coworkers [15].

\section{DYNAMICS}

As a simple but telling measure of structural relaxation, we focus our analysis here to a survey of singleparticle dynamics and monitor the mean square displacement (MSD) versus time. The MSD is defined via $\operatorname{MSD}\left(t, t_{0}\right)=$ $\frac{1}{N} \sum_{i=1}^{N}\left\langle\left[\mathbf{r}_{i}\left(t_{0}\right)-\mathbf{r}_{i}\left(t+t_{0}\right)\right]^{2}\right\rangle$, where $i$ is the index of a particle and $N$ the total number of particles. The brackets $\langle\cdot\rangle$ denotes statistical average over independent runs, where all the initial configurations are prepared in identical way using the same protocol. In this study, the initial time, $t_{0}$, is of great importance due to the loss of ergodicity upon the glass transition. If one chooses point Q (Fig. 4) as the origin of time, then $t_{0}$ becomes identical to the aging time.

As a reference for later comparisons, Fig. 5 illustrates the effect of aging in unperturbed samples for two different quench temperatures, both well in the glassy regime: $T_{0}=0.1$ $\left(\approx 0.25 T_{\mathrm{g}}\right)$ and $0.27\left(\approx 0.68 T_{\mathrm{g}}\right)$. As expected, the dynamics of a single particle shows a typical two-step relaxation behavior with an intermittent arrest in the cage made by the neighboring particles. This is visible in the horizontal segment (plateau) of the MSD curve, which extends to larger times upon aging. The life time of the nearest neighbor cage thus increases with $t_{\mathrm{w}}$. Not unexpectedly, Fig. 5 also reveals that, for the same aging time, a sample which lies deeper in the glass domain

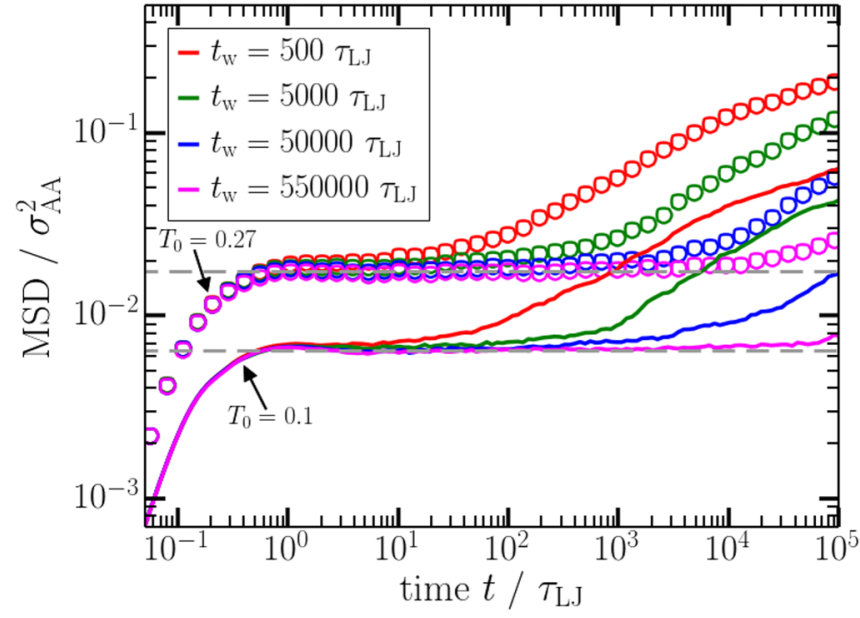

FIG. 5. Mean square displacements of A-particles versus time after being rapidly quenched to a temperature $T_{0}$ below the glass transition point of the model ( $T_{\mathrm{g}} \approx 0.41$ in reduced LJ units). Solid lines correspond to $T_{0}=0.1$ and symbols to $T_{0}=0.27$. For each $T_{0}$, the aging time increases from top to bottom with values as indicated. Aging proceeds faster (dynamics slows down more rapidly with $t_{\mathrm{w}}$ ) for a lower $T_{0}$. The gray dashed lines give the squared cage size $\xi$. Within harmonic approximation, the two cage sizes are related by the ratio of temperatures. This estimate is well satisfied by the present simulations (we find $\xi / T_{0} \approx 0.0645$ for all temperatures under investigation).

(i.e., is quenched to a lower temperature, $T_{0}$ ) shows a slower dynamics as compared to a less deeply quenched sample.

Figure 6 compares mean square displacements versus time obtained for a sample subject to constant-pressure cryogenic cycling (configuration $\mathrm{C}_{\mathrm{II}}$ in Fig. 4) with unperturbed samples which undergo aging alone for durations of $t_{\mathrm{w}}^{\prime}=t_{\mathrm{w}}+t_{\mathrm{tc}}$

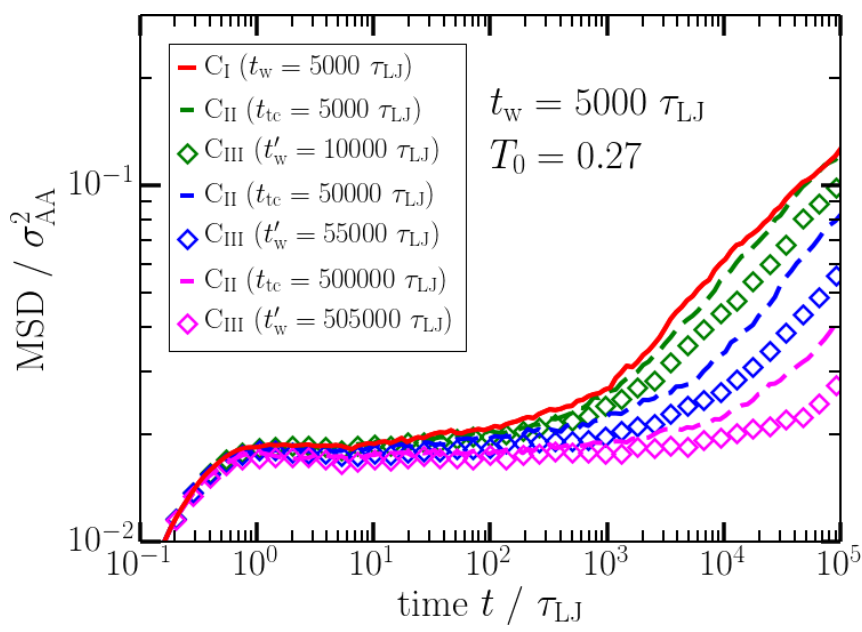

FIG. 6. Mean square displacements for a sample subject to thermal cycling (point $\mathrm{C}_{\mathrm{II}}$ in Fig. 4) and two noncycled ones (points $\mathrm{C}_{\mathrm{I}}$ and $\mathrm{C}_{\mathrm{III}}$ ) for $t_{\mathrm{w}}=5000 \tau_{\mathrm{LJ}}$ and different values of cycling time $t_{\mathrm{tc}}$ as indicated. Simulations of thermal cycling are performed at constant pressure $(\mathrm{NpT})$, but measurement of MSD at constant volume $(N V T)$. Apparently, thermal cycling slows down the aging process (compare $\mathrm{C}_{\mathrm{II}}$ and $\mathrm{C}_{\mathrm{III}}$ ) but does neither stop nor reverse this process $\left(\mathrm{C}_{\mathrm{I}}\right.$ and $\left.\mathrm{C}_{\mathrm{III}}\right)$. 


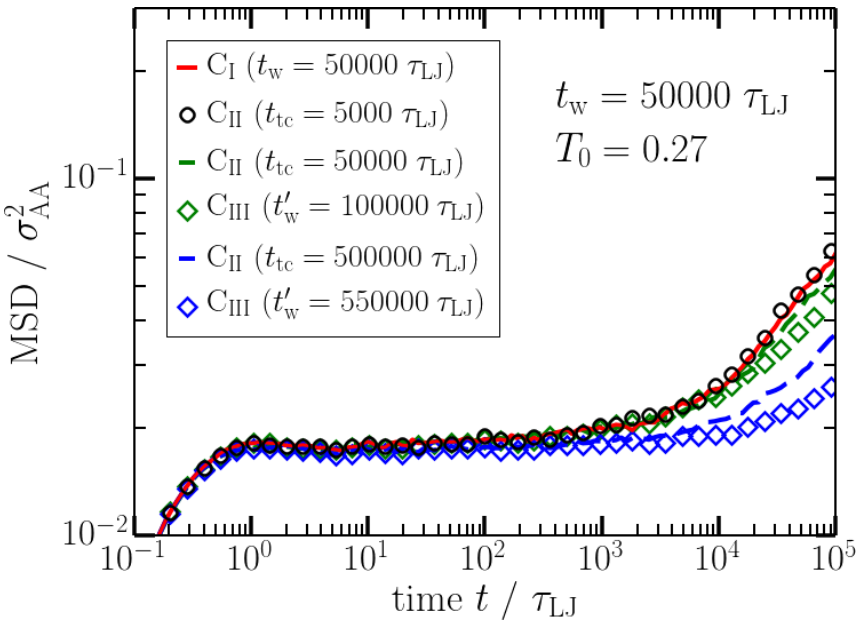

FIG. 7. The same type of data as in Fig. 6 but for a 10 times larger aging time of $t_{\mathrm{w}}=50000 \tau_{\mathrm{LJ}}$ showing that the behavior observed in Fig. 6 does not depend on $t_{\mathrm{w}}$. Furthermore it becomes evident that cycling times shorter than the initial aging time (black circles, $t_{\mathrm{tc}}=$ $5000 \tau_{\mathrm{LJ}}=0.1 t_{\mathrm{w}}$ ) do not lead to any differing observation, showing no impact of the thermal treatment on dynamics.

$\left(\mathrm{C}_{\mathrm{III}}\right)$ and $t_{\mathrm{w}}\left(\mathrm{C}_{\mathrm{I}}\right)$. The quenching temperature in this case is $T_{0}=0.27\left(\approx 0.68 T_{\mathrm{g}}\right)$, and the lower cycling temperature is $T_{\min }=0.07\left(\approx 0.18 T_{\mathrm{g}}\right)$. The figure contains data for three durations of thermal cycling, covering two decades in $t_{\mathrm{tc}}$ for a fixed initial aging time of $t_{\mathrm{w}}=5000 \tau_{\mathrm{LJ}}$. This serves to resolve the effects arising from aging as opposed to those of temperature variations. Based on the data shown in Fig. 6, the following observations can be made. If the measurement of dynamic quantities starts at the same time instant for all samples (configurations $\mathrm{C}_{\mathrm{II}}$ and $\mathrm{C}_{\mathrm{III}}$ ), the ones subject to cryogenic cycling exhibit a faster dynamics as compared to the unperturbed. However, if one compares the system dynamics prior to temperature variations (i.e., if one starts to measure MSD from point $C_{I}$ onward) with those at the end of the thermal treatment $\left(\mathrm{C}_{\mathrm{II}}\right)$, a slowing down of the structural relaxation is observed.

To check possible effects of the initial aging process, we have varied $t_{\mathrm{w}}$ within two decades in time, $t_{\mathrm{w}} \in$ $\{500,5000,50000\} \tau_{\mathrm{LJ}}$. As shown in Fig. 7 for $t_{\mathrm{w}}=$ $50000 \tau_{\mathrm{LJ}}$, this behavior does not change if one varies the time between temperature quench and the beginning of thermal cycling. However, one should keep in mind that, to resolve the effect of decelerated aging dynamics during thermal cycling in an unambiguous way, $t_{\mathrm{tc}}$ must be large compared to $t_{\mathrm{w}}$. This aspect makes simulation studies of larger $t_{\mathrm{w}}$ computationally demanding.

An aspect in the context of cryogenic cycling regards the maximum and minimum temperatures achieved during the process. In fact, for rejuvenation to become effective, it is expected that the temperature shall be low enough so that stresses, which are induced during repeated cooling and reheating due to medium range heterogeneities in thermal expansion coefficients, appear steady [14] within the time scale of heat treatment but enhance the dynamics of slow processes which determine the system response to a mechanical perturbation (and thus are responsible for mechanical properties
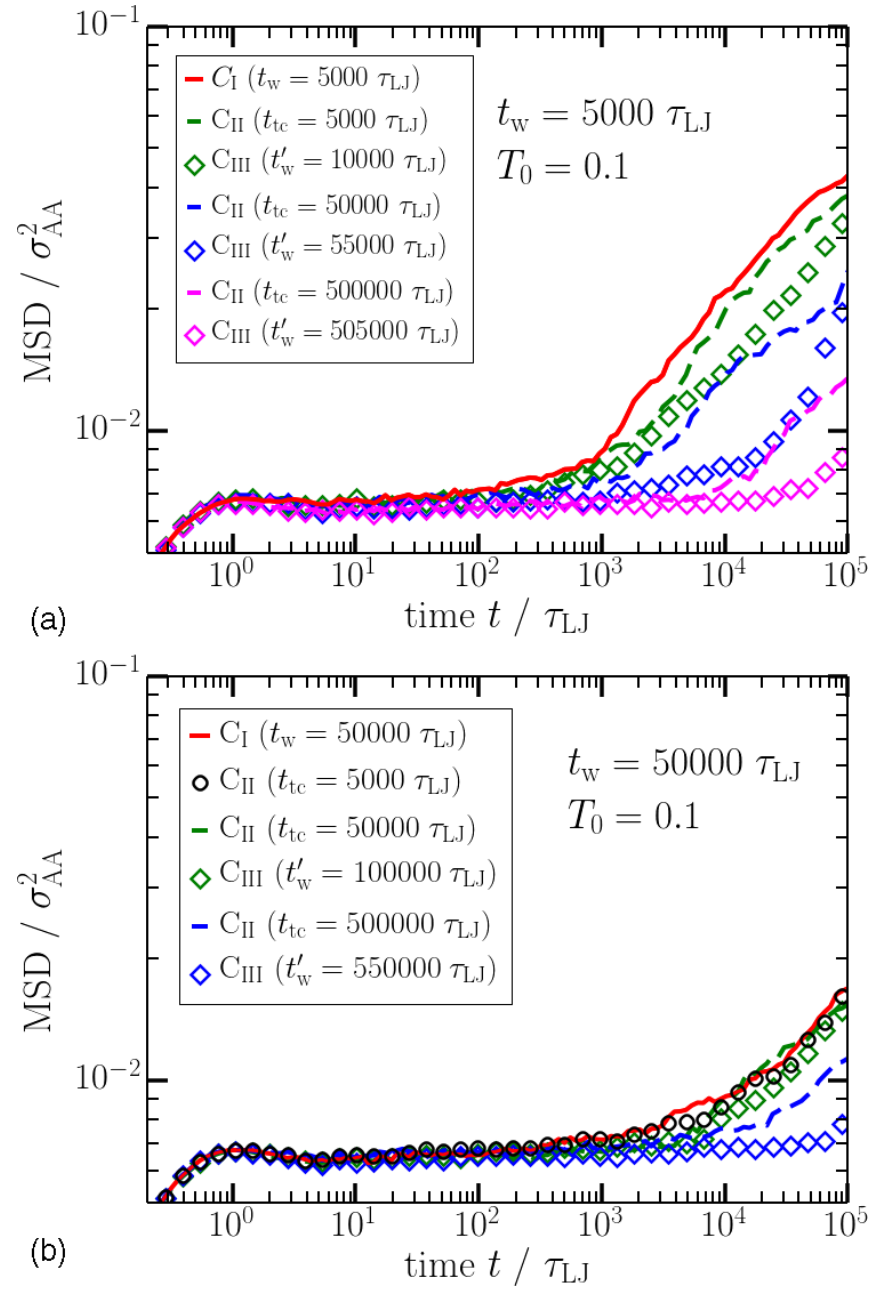

FIG. 8. The same type of data as in Fig. 6 but for a quench temperature of $T_{0}=0.1$. The same trends as in the case of $T_{0}=0.27$ are observed.

at low frequencies). Therefore, we check below whether the subdominant character of rejuvenation in the above described simulations is due to not sufficiently low temperatures. For this purpose, we have prepared samples at $T_{0}=0.1 \approx 0.25 T_{\mathrm{g}}$ and have performed thermal cycling between this temperature and $T_{\min }=0.0001$. As seen from Fig. 8, the same trends are observed as in the case of higher cycling temperature of $T_{0}=0.27$ and $T_{\min }=0.07$.

In order to check the possible impact of system size [15], we performed simulations of a much larger system containing around 1.2 million particles. The sample is quenched to $T_{0}=0.2$ and has been subject to thermal cycling between this temperature and $T_{\min }=0.01$. As shown in Fig. 9, in full agreement with results obtained for the smaller system, cryogenic cycling decelerates aging but does not stop it, nor reverses the trend towards a rejuvenation.

The above observations can be summarized as follows. First, as expected, in a liquid rapidly quenched to a low temperature below $T_{\mathrm{g}}$, physical aging is always present. Second, the dynamics of aging is slower if the system undergoes deep thermal cycling. From this, one can directly conclude that rejuvenation processes are triggered via thermal cycling. 


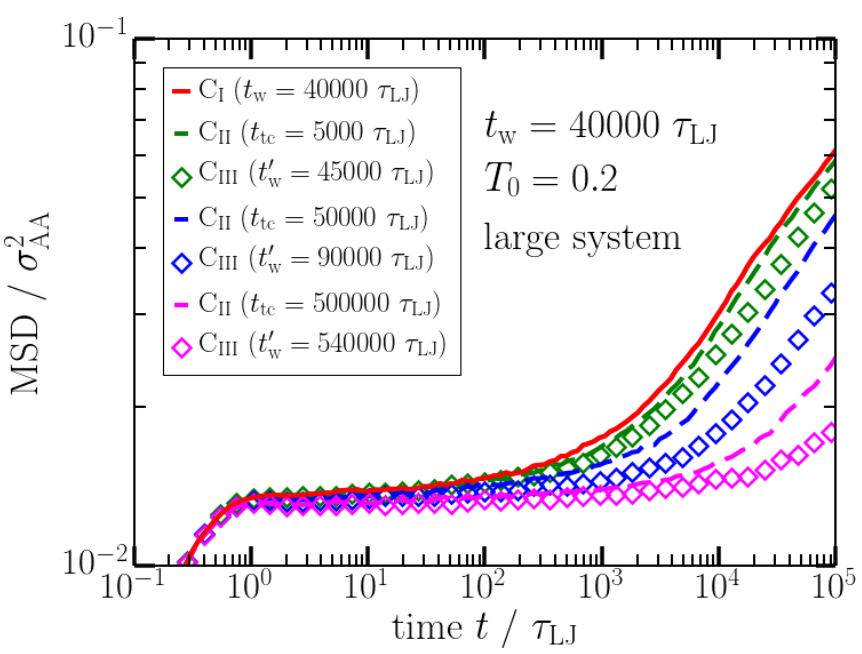

FIG. 9. The same type of data as in Fig. 6 and 8 but for a large system of 1.2 million particles and a quench temperature of $T_{0}=0.2$. The same trends as in the case of smaller systems are observed.

The fact that the overall dynamics of relaxation is dominated by aging indicates that a potential rejuvenation effect is not strong enough to stop or reverse the evolution of the system towards equilibrium.

\section{ENERGY DISTRIBUTION}

As a result of the evolution towards metastable equilibrium, the free energy decreases during the aging process. In many systems such as the present binary LJ glass, also the potential energy decreases during aging. Moreover, the potential energy distribution becomes narrower as time proceeds. A rejuvenation process is thus expected to lead to the contrary effect, i.e., increasing the potential energy and broadening its distribution [34]. This provides an alternative test of the possible rejuvenation effects due to thermal cycling.

However, Fig. 10(a) shows that, due to the binary character of the present model, the energy distribution has a bimodal shape. This feature complicates a quantitative analysis of aging effect as it is not straight forward to define a width for such a distribution function. Interestingly, as also shown in Fig. 10(a), if one evaluates the energy per particle for A- and B-species separately, the corresponding probability densities are unimodal. For each species, one can then follow the evolution of the variance (squared characteristic width) of the distribution function both upon unperturbed aging as well after thermal cycling. Indeed, in line with the above observations of dynamics, the distribution of energy becomes narrower both upon aging and thermal cycling with increasing age and total cycling time, respectively [Fig. 10(b) and 11(a)]. Moreover, this process is less pronounced upon thermal cycling, thus signaling again a weakening of aging effects. In full agreement with this behavior, average potential energy continues decreasing with $t_{\mathrm{tc}}$, but slower than upon pure aging [Fig. 11(b)].

Again, we do the same comparison of results after thermal cycling and after only-aging for a larger system of 1.2 million atoms. As can be seen from Fig. 12 one finds similar trends as in the case of a small system.
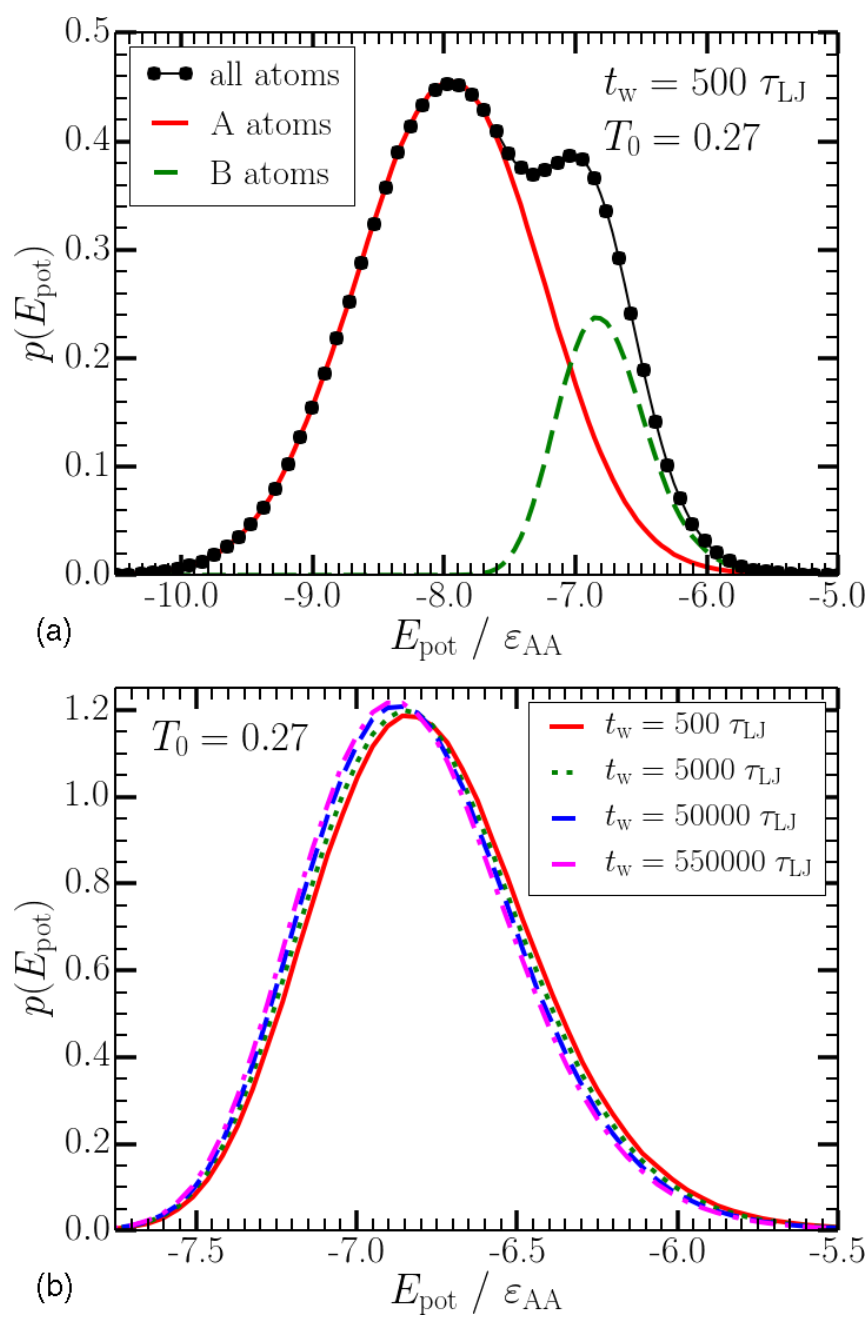

FIG. 10. Probability density for potential energy per particle. (a) The dotted line corresponds to total energy and shows a bimodal shape, characteristic of a binary mixture. Solid and dashed lines show the energy distribution of each individual species A and B, respectively. Distributions are normalized for the total number of particles in the system, $N$. (b) Distribution of potential energy per particle for B-atoms evaluated after different aging times $t_{\mathrm{w}}$. A narrowing of the distribution can be inferred from this plot as well as a shift of the mean energy level towards lower potential energy values. Distributions are normalized for the total number of B-atoms in the system, $N_{\mathrm{B}}$.

\section{FREQUENCY VERSUS CYCLING TIME}

Interestingly, the results obtained thus far do not depend much on the specific cycling frequency. Rather, they are sensitive to the total duration of thermal cycling. To underline this issue, Fig. 13 shows variances of potential energy distributions after thermal cycling relative to the variance of the initial configuration (red plus). All samples have been cycled for the same duration $t_{\mathrm{tc}}=$ $50000 \tau_{\mathrm{LJ}}$ but with different frequencies, thus holding times $t_{\mathrm{h}} \in\left\{12.5 \tau_{\mathrm{LJ}}, 125 \tau_{\mathrm{LJ}}, 1250 \tau_{\mathrm{LJ}}\right\}$ and number of cycles, $n \in$ $\{1000,100,10\}$. Although one can observe a difference in the resulting potential energy variances, the maximum difference of variances after cycling (between the cases 

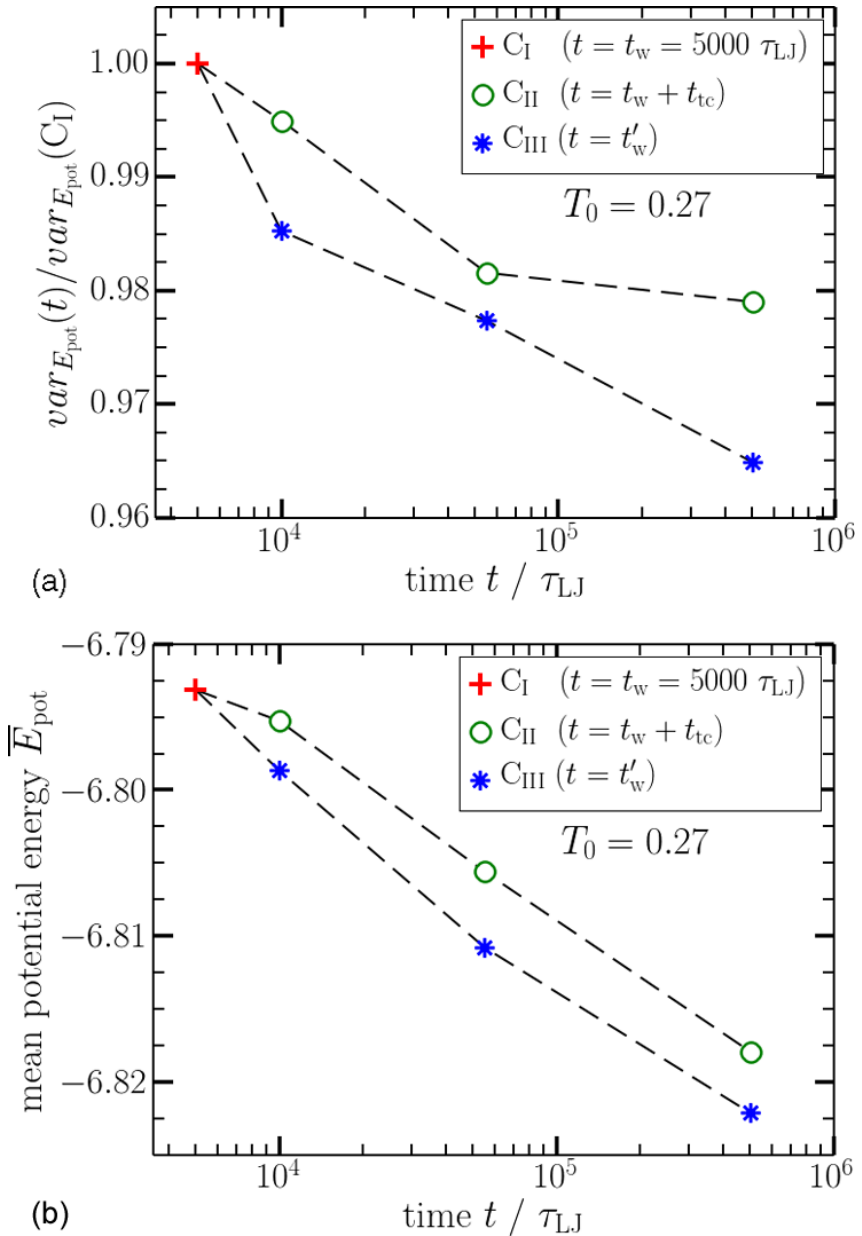

FIG. 11. Effect of thermal cycling on the variance (a) and level (b) of potential energy of a B-particle. The red plus is the initial configuration at point $\mathrm{C}_{\mathrm{I}}$. Note that this sample has already undergone aging during a time of $t_{\mathrm{w}}=5000 \tau_{\mathrm{LJ}}$. The blue stars correspond to unperturbed samples undergoing aging alone $\left(\mathrm{C}_{\mathrm{III}}\right)$. In this case, the time axis gives the aging time, $t \equiv t_{\mathrm{w}}^{\prime}$. The green circles are results of thermal treatment. Here, the horizontal axis stands for the total duration of TC plus an offset of the initial aging time, $t \equiv t_{\mathrm{tc}}+t_{\mathrm{w}}$. In both cases, variance as well as the mean potential energy decrease, signaling that the thermal treatment does not stop aging.

of $t_{\mathrm{h}}=12.5 \tau_{\mathrm{LJ}}$, green circle, and $t_{\mathrm{h}}=1250 \tau_{\mathrm{LJ}}$, magenta diamond) is $0.37 \% \operatorname{var}_{E_{\mathrm{pot}}}\left(\mathrm{C}_{\mathrm{I}}\right)$. Comparing to the previous cases with a constant number of cycles but a varying thermal cycling time, where we find differences of 1 to $2 \% \operatorname{var}_{E_{\mathrm{pot}}}\left(\mathrm{C}_{\mathrm{I}}\right)$, we conclude that the impact of thermal cycling time is much greater than the impact of frequency.

\section{CONCLUSION AND OUTLOOK}

The present study is motivated by recent reports on improved plasticity of bulk metallic glasses upon deeply cooled (cryogenic) thermal cycling $[10,17]$. It has been argued that, since metallic glasses have a nonuniform structure, the local heterogeneity of the thermal expansion coefficient induces nonaffine strains during cycling from room temperature down to cryogenic temperatures that could accumulate and increase structural disorder [14].
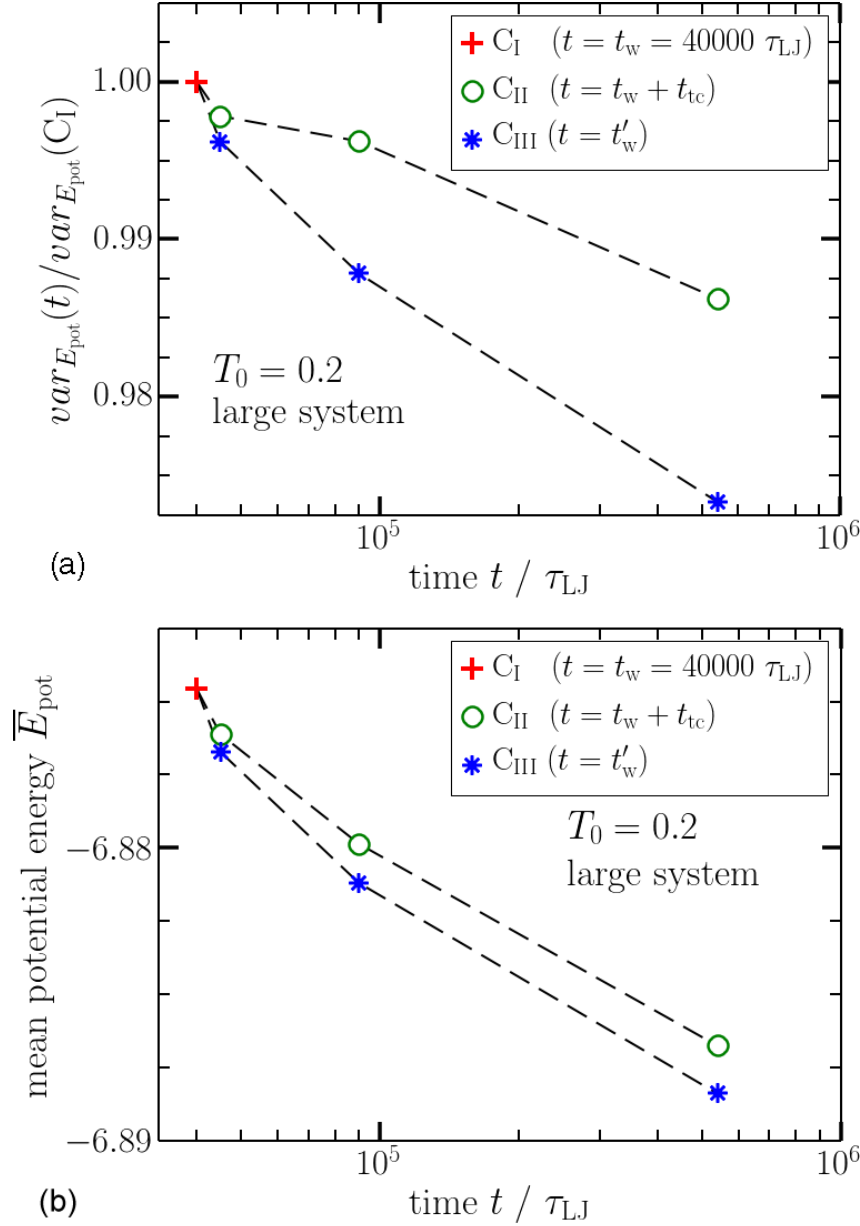

FIG. 12. The same type of data as in Fig. 11 but for a large system containing 1.2 million particles. For the variance of potential energy distribution as well as for the mean potential energy we find the same trends as observed for the smaller system.

The experimental part of this work shows the results of DSC measurements on $\mathrm{Pd}_{40} \mathrm{Ni}_{40} \mathrm{P}_{20}, \mathrm{Pd}_{39} \mathrm{Ni}_{41} \mathrm{P}_{20}$ and $\mathrm{Zr}_{41.2} \mathrm{Be}_{22.5} \mathrm{Ti}_{13.8} \mathrm{Cu}_{12.5} \mathrm{Ni}_{10}$ metallic glasses with and without cryogenic thermal cycling. Samples are investigated in their as-quenched state and after preannealing below $T_{\mathrm{g}}$ introducing a structural relaxation of the as-quenched structure. Despite other reports, our DSC results suggest that, for the good glass formers investigated here, structural disorder does not increase sufficiently high in order to relax by heating and to induce a measurable heat signal.

With an increasing number of thermal cycles, a change of the total heat release below $T_{\mathrm{g}}, \Delta H_{\text {rel }}$, is observed. Whereas in PdNiP the value of $\Delta H_{\text {rel }}$ is decreasing for a low number of cycles, it slightly increases in Zr-based glass with respect to the corresponding as-quenched levels. However, after at least 20 cycles, the total heat release approaches the values of $\Delta H$ measured for as-quenched glasses. The calorimetric response of PdNiP and Zr-based bulk metallic glasses on cryogenic thermal cycling thus seems to be independent on the level of excess free volume.

Both in as-quenched samples as well as after their full relaxation, no significant rejuvenation effect is observed. Since structural fluctuations are at heart of TC-induced rejuvenation 


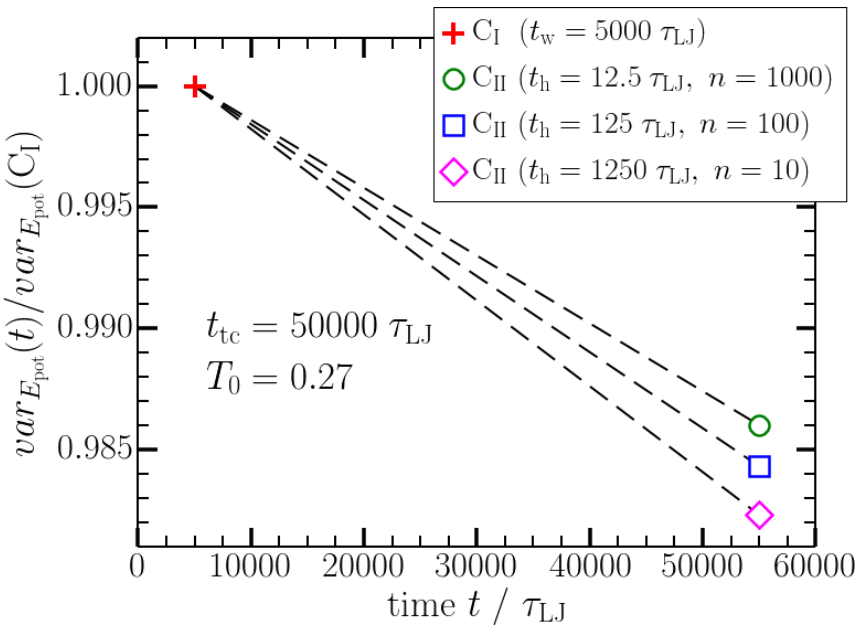

FIG. 13. Variances of potential energy distributions of B-atoms before (configuration $\mathrm{C}_{\mathrm{I}}$, red plus) and after thermal cycling. The samples have been aged for $t_{\mathrm{w}}=5000 \tau_{\mathrm{LJ}}$. All samples denoted as $\mathrm{C}_{\mathrm{II}}$ have been cycled for the same duration $t_{\mathrm{tc}}=50000 \tau_{\mathrm{LJ}}$ but with different frequencies by varying holding times, $t_{\mathrm{h}} \in$ $\{12.5,125,1250\} \tau_{\mathrm{LJ}}$. This leads to a variation of the total number of thermal cycles, $n \in\{1000,100,10\}$. The maximum difference of variances after cycling (between the cases of $t_{\mathrm{h}}=12.5 \tau_{\mathrm{LJ}}$, green circle, and $t_{\mathrm{h}}=1250 \tau_{\mathrm{LJ}}$, magenta diamond) is $0.37 \% \operatorname{var}_{E_{\mathrm{pot}}}\left(\mathrm{C}_{\mathrm{I}}\right)$.

effects, these findings suggest a low degree of heterogeneity in our samples, which are excellent glass formers. This calls for future studies of structure in materials with good glassforming capability.

The second part of this work presents a detailed investigation of single particle dynamics and energy distribution in a binary Lennard Jones glass subject to oscillatory variations of temperature far below the glass transition. The choice of this generic model is motivated by the aim to focus on generic rather than materials specific aspects. Through comparisons with the same system subject to aging at constant temperature, we find that aging is slowed down but not stopped neither reversed during thermal treatment.

Specifically, upon increasing the duration of cryogenic cycling, dynamics continues to slow down, the average interaction energy decreases and the corresponding distribution function becomes narrower. All these processes occur also upon simple aging but are more enhanced as compared to thermally cycled configurations. From this comparison, we conclude that TC-induced rejuvenation plays a subdominant role as compared to physical aging.

As mentioned in the introduction, rejuvenation effects induced by deep thermal cycling are presumed to be stronger in more heterogeneous glasses [14]. In this context, it is noteworthy that an instantaneous quench to the glassy state creates a strongly heterogeneous structure in the present model [36]. Despite this strong quench, TC-induced rejuvenation remains sub-dominant compared to aging.

These observations call for future studies on the subject in order to identify the mechanisms which may lead to improved ductility in metallic glasses. In this context, it would be helpful to explore changes to the structure and dynamics within different stages of a single temperature cycle [53]. Another challenging question here is how to increase structural heterogeneity without deteriorating mechanical properties such as propensity to shear band formation [36]. Last but not least the possible connection between glass-forming ability and structural homogeneity deserves a thorough investigation.

\section{ACKNOWLEDGMENTS}

We are grateful to Alexandra Lagogianni and Harald Rösner for fruitful discussions. Financial support by the Deutsche Forschungsgemeinschaft (DFG, German Research Foundation) under the project numbers VA205/16-1 and WI $1899 / 29-1$ is acknowledged.
[1] L. Struik, Physical Aging in Amorphous Polymers and Other Materials (Elsevier Science, Delft, Netherlands, 1978).

[2] W. Kob and J.-L. Barrat, Aging Effects in a Lennard-Jones Glass, Phys. Rev. Lett. 78, 4581 (1997).

[3] N. H. Siboni, D. Raabe, and F. Varnik, Aging in amorphous solids: A study of the first-passage time and persistence time distributions, Europhys. Lett. 111, 48004 (2015).

[4] D. J. A. Senden, J. A. W. van Dommelen, and L. E. Govaert, Physical aging and deformation kinetics of polycarbonate, J. Polymer Sci. Part B: Polymer Phys. 50, 1589 (2012).

[5] C. A. Pampillo, English Flow and fracture in amorphous alloys, J. Mater. Sci. 10, 1194 (1975).

[6] A. Argon, Mechanisms of inelastic deformation in metallic glasses, J. Phys. Chem. Solids 43, 945 (1982).

[7] H. van Melick, L. Govaert, and H. Meijer, Localisation phenomena in glassy polymers: Influence of thermal and mechanical history, Polymer 44, 3579 (2003).
[8] C. A. Schuh, T. C. Hufnagel, and U. Ramamurty, Mechanical behavior of amorphous alloys, Acta Mater. 55, 4067 (2007).

[9] M. Ozawa, L. Berthier, G. Biroli, A. Rosso, and G. Tarjus, Random critical point separates brittle and ductile yielding transitions in amorphous materials, Proc. Natl. Acad. Sci. USA 115, 6656 (2018).

[10] S. V. Ketov, Y. H. Sun, S. Nachum, Z. Lu, A. Checchi, A. R. Beraldin, H. Y. Bai, W. H. Wang, D. V. Louzguine-Luzgin, M. A. Carpenter, and A. L. Greer, Rejuvenation of metallic glasses by non-affine thermal strain, Nature (London) 524, 200 (2015).

[11] M. Wakeda, J. Saida, J. Li, and S. Ogata, Controlled rejuvenation of amorphous metals with thermal processing, Sci. Rep. 5, 10545 (2015).

[12] J. Saida, R. Yamada, M. Wakeda, and S. Ogata, Thermal rejuvenation in metallic glasses, Sci. Techn. Adv. Mater. 18, 152 (2017). 
[13] N. Miyazaki, M. Wakeda, Y.-J. Wang, and S. Ogata, Prediction of pressure-promoted thermal rejuvenation in metallic glasses, npj Comput. Mater. 2, 16013 (2016).

[14] T. C. Hufnagel, Metallic glasses: Cryogenic rejuvenation, Nat. Mater. 14, 867 (2015).

[15] B. Shang, P. Guan, and J.-L. Barrat, Role of thermal expansion heterogeneity in the cryogenic rejuvenation of metallic glasses, J. Phys.: Mater. 1, 015001 (2018).

[16] N. V. Priezjev, The effect of cryogenic thermal cycling on aging, rejuvenation, and mechanical properties of metallic glasses, J. Non-Cryst. Solids 503-504, 131 (2019).

[17] W. Guo, R. Yamada, and J. Saida, Rejuvenation and plasticization of metallic glass by deep cryogenic cycling treatment, Intermetallics 93, 141 (2018).

[18] W. Guo, J. Saida, M. Zhao, S. Lü, and S. Wu, Unconspicuous rejuvenation of a Pd-based metallic glass upon deep cryogenic cycling treatment, Mater. Sci. Eng.: A 759, 59 (2019).

[19] G. Wilde, G. Görler, R. Willnecker, and G. Dietz, Thermodynamic properties of $\mathrm{Pd}_{40} \mathrm{Ni}_{40} \mathrm{P}_{20}$ in the glassy, liquid, and crystalline states, Appl. Phys. Lett. 65, 397 (1994).

[20] G. Wilde, G. Görler, R. Willnecker, and H. Fecht, Calorimetric, thermomechanical, and rheological characterizations of bulk glass-forming $\mathrm{Pd}_{40} \mathrm{Ni}_{40} \mathrm{P}_{20}$, J. Appl. Phys. 87, 1141 (2000).

[21] Y. Mitrofanov, M. Peterlechner, I. Binkowski, M. Zadorozhnyy, I. Golovin, S. Divinski, and G. Wilde, The impact of elastic and plastic strain on relaxation and crystallization of Pd-Ni-P-based bulk metallic glasses, Acta Mater. 90, 318 (2015).

[22] H. Zhou, R. Hubek, M. Peterlechner, and G. Wilde, Two-stage rejuvenation and the correlation between rejuvenation behavior and the boson heat capacity peak of a bulk metallic glass, Acta Mater. 179, 308 (2019).

[23] R. Hubek, M. Seleznev, I. Binkowski, M. Peterlechner, S. Divinski, and G. Wilde, The impact of micro-alloying on relaxation dynamics in $\mathrm{Pd}_{40} \mathrm{Ni}_{40} \mathrm{P}_{20}$ bulk metallic glass, J. Appl. Phys. 124, 225103 (2019).

[24] S. Hilke, H. Rösner, D. Geissler, A. Gebert, M. Peterlechner, and G. Wilde, The influence of deformation on the mediumrange order of a $\mathrm{Zr}$-based bulk metallic glass characterized by variable resolution fluctuation electron microscopy, Acta Mater. 171, 275 (2019).

[25] X. Bian, G. Wang, J. Yi, Y. Jia, J. Bednarčík, Q. Zhai, I. Kaban, B. Sarac, M. Mühlbacher, F. Spieckermann, J. Keckes, and J. Eckert, Atomic origin for rejuvenation of a Zr-based metallic glass at cryogenic temperature, J. Alloys Compd. 718, 254 (2017).

[26] W. Guo, R. Yamada, J. Saida, S. Lü, and S. Wu, Various rejuvenation behaviors of zr-based metallic glass by cryogenic cycling treatment with different casting temperatures, Nanoscale Res Lett. 13, 398 (2018).

[27] W. Kob and H. C. Andersen, Scaling Behavior in the $\beta$ Relaxation Regime of a Supercooled Lennard-Jones Mixture, Phys. Rev. Lett. 73, 1376 (1994).

[28] W. Kob and H. C. Andersen, Testing mode-coupling theory for a supercooled binary Lennard-Jones mixture I: The van Hove correlation function, Phys. Rev. E 51, 4626 (1995).

[29] W. Kob and H. C. Andersen, Testing mode-coupling theory for a supercooled binary Lennard-Jones mixture II: Intermediate scattering function and dynamic susceptibility, Phys. Rev. E 52, 4134 (1995).
[30] P. Scheidler, W. Kob, and K. Binder, The relaxation dynamics of a simple glass former confined in a pore, Europhys. Lett. 52, 277 (2000).

[31] P. Scheidler, W. Kob, and K. Binder, Cooperative motion and growing length scales in supercooled confined liquids, Europhys. Lett. 59, 701 (2002).

[32] P. Scheidler, W. Kob, and K. Binder, The relaxation dynamics of a supercooled liquid confined by rough walls, J. Phys. Chem. B 108, 6673 (2004).

[33] F. Varnik, L. Bocquet, J.-L. Barrat, and L. Berthier, Shear Localization in a Model Glass, Phys. Rev. Lett. 90, 095702 (2003).

[34] F. Varnik, L. Bocquet, and J.-L. Barrat, A study of the static yield stress in a binary Lennard-Jones glass, J. Chem. Phys. 120, 2788 (2004).

[35] F. Varnik, Structural relaxation and rheological response of a driven amorphous system, J. Chem. Phys. 125, 164514 (2006).

[36] M. Hassani, A. E. Lagogianni, and F. Varnik, Probing the Degree of Heterogeneity Within a Shear Band of a Model Glass, Phys. Rev. Lett. 123, 195502 (2019).

[37] I. Binkowski, G. Shrivastav, J. Horbach, S. Divinski, and G. Wilde, Shear band relaxation in a deformed bulk metallic glass, Acta Mater. 109, 330 (2016).

[38] N. Nollmann, Plastische Deformation und mechanische Eigenschaften von Palladium basierten metallischen Gläsern, Ph.D. thesis, Münster University, 2014.

[39] J. Bünz and G. Wilde, Direct measurement of the kinetics of volume and enthalpy relaxation of an Au-based bulk metallic glass, J. Appl. Phys. 114, 223503 (2013).

[40] J. Lübke, Cryogenic cycling of bulk metallic glasses, Ph.D. thesis, Münster University, 2017.

[41] A. Das, E. Dufresne, and R. Maaß, Structural dynamics and rejuvenation during cryogenic cycling in a Ar-based metallic glass, Acta Mater. 196, 723 (2020).

[42] J. Löffler and W. Johnson, Model for decomposition and nanocrystallization of deeply undercooled $\mathrm{Zr}_{41.2} \mathrm{Ti}_{13.8} \mathrm{Cu}_{12.5} \mathrm{Ni}_{10} \mathrm{Be}_{22.5}$, Appl. Phys. Lett. 76, 3394 (2000).

[43] R. Busch, Z. Evenson, I. Gallino, and S. Wei, Thermodynamics, kinetics and fragility of bulk metallic glass forming liquids, arXiv:1405.2251.

[44] J. P. Wittmer, H. Xu, P. Polińska, F. Weysser, and J. Baschnagel, Shear modulus of simulated glass-forming model systems: Effects of boundary condition, temperature, and sampling time, J. Chem. Phys. 138, 12A533 (2013).

[45] F. Varnik and O. Henrich, Yield stress discontinuity in a model glass, Phy. Rev. B 73, 174209 (2006).

[46] M. Hassani, P. Engels, D. Raabe, and F. Varnik, Localized plastic deformation in a model metallic glass: A survey of free volume and local force distributions, J. Stat. Mech. (2016) 084006.

[47] M. Hassani, P. Engels, and F. Varnik, Wall effects on spatial correlations of non-affine strain in a 3D model glass, Europhys. Lett. 121, 18005 (2018).

[48] M. Hassani, E. M. Zirdehi, K. Kok, P. Schall, M. Fuchs, and F. Varnik, Long-range strain correlations in 3D quiescent glass forming liquids, Europhys. Lett. 124, 18003 (2018).

[49] S. Plimpton, Fast parallel algorithms for short-range molecular dynamics, J. Comput. Phys. 117, 1 (1995). 
[50] W. G. Hoover, Canonical dynamics: Equilibrium phase-space distributions, Phys. Rev. A 31, 1695 (1985).

[51] M. Parrinello and A. Rahman, Polymorphic transitions in single crystals: A new molecular dynamics method, J. Appl. Phys. 52, 7182 (1981).
[52] F. Varnik, J. Baschnagel, and K. Binder, Reduction of the glass transition temperature in polymer films: A molecular-dynamics study, Phy. Rev. E 65, 021507 (2002).

[53] C. Scalliet and L. Berthier, Rejuvenation and Memory Effects in a Structural Glass, Phys. Rev. Lett. 122, 255502 (2019). 